\title{
Données Préliminaires Sur La Carotte M2005-12 Prélevée Au Sommet De L'escarpement Pen Duick (Champ De Volcans De Boue D'el Araich, Maroc)
}

\author{
Adil Chiguer, PhD \\ Université Mohammed V- Agdal, Rabat, \\ Faculté des Sciences, Département des Sciences de la Terre, Maroc
}

doi: 10.19044/esj.2017.v13n27p201 URL:http://dx.doi.org/10.19044/esj.2017.v13n27p201

\begin{abstract}
The Pen Duick Escarpment is located 30 nautical miles off shore the Moroccan coast in the Gulf of Cadiz and is a fault formed in a compressional system. The area surrounding the escarpment is characterised by a very complex geological. This project aims to study the sedimentological processes on top of the Pen Duick Escarpment and relate them to changes of the last glacial-interglacial period. For this purpose, the sediments were analysed using the X-ray fluorescence (XRF), grain-size distribution and the stable oxygen isotope measurements of planktonic foraminifera. Analysis of the core shows that the core consists mainly of silty clay with foraminifera. Several sandy layers were observed in the core at different depths. In some zones,coral fragments occur, which seem to have been transported to the core site. Stable isotope analysis of planktonic foraminifera shows a repetition of recent Holocene material in the pistoncore. The changes in composition and mineralogy of the sediment in the core might be related to sedimentological processes like currents of strong energy.
\end{abstract}

Keywords: Pen Duick Escarpment, mud volcano, planktonic foraminifera, oxygen isotopes

\section{Résumé}

L'Escarpement Pen Duick est situé à 30 milles marins de la côte marocaine dans la partie méridionale de Golfe de Cadix; c'est une ride soulevée par une faille dans un système compressif. Le secteur qui l'entoure est caractérisé par une histoire géologique complexe. Le but principal de ce travail est d'étudier les processus sédimentologiques au sommet de l'Escarpement Pen Duick et leurs relations avec les changements climatiques des dernières périodes glaciaires et interglaciaires. A cet effet, les sédiments ont été analysés par la fluorescence de rayon X (XRF), par la granulométrie 
et grâce à l'étude des isotopes stables d'oxygène pour les foraminifères planctoniques. La carotte montre une dominance de la composition silto argileuse avec la présence des foraminifères. On a observé aussi l'emplacement de sable dans certaines profondeurs de la carotte. Dans quelques zones, des fragments de coraux sont présents. L'analyse des isotopes stables pour les foraminifères planctoniques indique une répétition de matériel daté d'Holocène récent. Les changements de la composition sédimentaire et minéralogique trouvés dans la carotte pourraient être liés aux processus sédimentologiques comme les courants de forte énergie.

Mots-clés: Escarpement Pen Duick, volcans de boue, foraminifères planctoniques, isotopes d'oxygène

\section{Introduction}

Le Golfe de Cadix est situé à l'Ouest du Détroit de Gibraltar, entre les côtes sud-ouest de l'Ibérie et celles du Nord -ouest du Maroc, dans la frontière des plaques africaine et ibérique (Maldonado et al. 1999). Sa structure est dominée par un prisme sédimentaire en lobe (Maldonado et al. 1999, Medialdea et al. 2004) de dépôts d'âge allant du Trias au Quaternaire. Ce prisme comprend dans sa partie supérieure une unité de dépôt à signature chaotique (unité U4; Medialdea et al. 2004), qualifiée d' « olistostrome » (Maldonado et al. 1999) et dénommée «Unité allochtone du Golfe de Cadix » (Allochtonous Unit of the Gulf of Cadiz; Medialdea et al. 2004). Cette unité d'âge Tortonien est épaisse de $2,7 \mathrm{~km}$ au maximum et serait issue de l'érosion de la chaîne bético-rifaine (Maldonado et al. 1999, Medialdea et al. 2004, Pinheiro et al. 2003, Stadnitskaia et al. 2006). Elle s'avancerait sur le plancher océanique atlantique depuis le Miocène tardif (10 Ma) (Maldonado et al. 1999, pinherio et al. 2003, Samoza et al, 2003).

La partie orientale du prisme qui est rattachée au domaine béticorifain offshore (Medialdea et al. 2004), est perforée par de nombreux volcans de boue. Au cours de la dernière décennie, ces volcans ont fait l'objet de nombreuses campagnes océanographiques internationales suite à la découverte d'émanations de gaz (Pinheiro et al. 2003, Stadniskaia et al. 2006). Les diverses études ont porté sur plusieurs aspects tels que l'hydrodynamisme, la sédimentologie, la structure, l'environnement, la biochimie et les ressources minérales et en hydrocarbures (Kenyon et al. 2003, Hamoumi et al. 2005). Parmi ces campagnes, on cite celle au Sud du Golfe de Cadix au large de Larache. Celle-ci a été financée par le projet Moundforce de la Fondation européenne de la science, qui fait partie du programme EUROMARGIN en vue de réaliser une étude sédimentologique et paléo-océanographique du sommet de l'Escarpement Pen Duick. Le but de ce ravail lors de cette mission était d'étudier les caractères physiques des 
dépôts et l'évolution des niveaux sédimentologiques du sommet de l'escarpement et leurs relations avec les changements climatiques des dernières périodes glaciaire-interglaciaires.

\section{Cadre Géologique}

L'Escarpement Pen Duick est situé au sein du champ de volcans de boue de Larache (El Arraich mud volcano field). Ce dernier est l'un des cinq champs de volcans de boue (GDR, DPM, TASYO, SPM et El Arraich) détectés dans le Golfe de Cadix (Van Rensbergen et al. 2003). La morphologie du champ d'El Arraich, décrite en détail par Van Rensbergen et al. (2003, 2005), comporte plusieurs volcans de boue (Gemini, Al Idrissi, Fiuza, Mercator...), des rides orientées WNW-ESE (Renard, Vernadsky) décalées au milieu selon un jeu dextre et un Escarpement Pen Duick. Ce dernier situé à 30 milles $(55,56 \mathrm{~km})$ marins de la côte avec $525 \mathrm{~m}$ de profondeur (de Haas 2006) correspond à une ride de direction NW-SE d'environ $4,5 \mathrm{~km}$ de longueur liée à une faille formée dans un système décrochant.

L'interprétation du Multibeam bathymétrique et de la sismique (Foubert et al. 2008) le long de l'Escarpement Pen Duick, montre que la ride est délimitée par une falaise dont la hauteur est de $100 \mathrm{~m}$ (65 dans la partie sud-ouest) et une pente de 15 à $20^{\circ}$. Dans la partie Est de la falaise, le gradient atteint $25^{\circ}$ (Foubert et al. 2008). Au sommet de la falaise, 15 monticules ont été identifiés entre 500 et $600 \mathrm{~m}$ de profondeur d'eau, ces monticules peuvent atteindre $60 \mathrm{~m}$ de hauteur. Ils sont allongés suivant la direction E-W sur une longueur d'environ un demi-kilomètre. À la base de la falaise, les fragments de petits monticules, qui ont été trouvés sont caractérisés par une direction E-W. La région de Pen Duick est caractérisée par des croûtes de carbonate liées aux infiltrations qui pourraient produire un substrat approprié pour la décantation de corail (Van Rooij et al. 2011)

Par intégration de la bathymétrie, des données sismiques et des images de sonar, 15 monticules ont été identifiés. Deux types de monticules ont été identifiés; les uns sont de forme allongée, les autres en grappes à cônes arrondis (fig.1\&2) (de Hass. 2006, Foubert et al. 2008). 


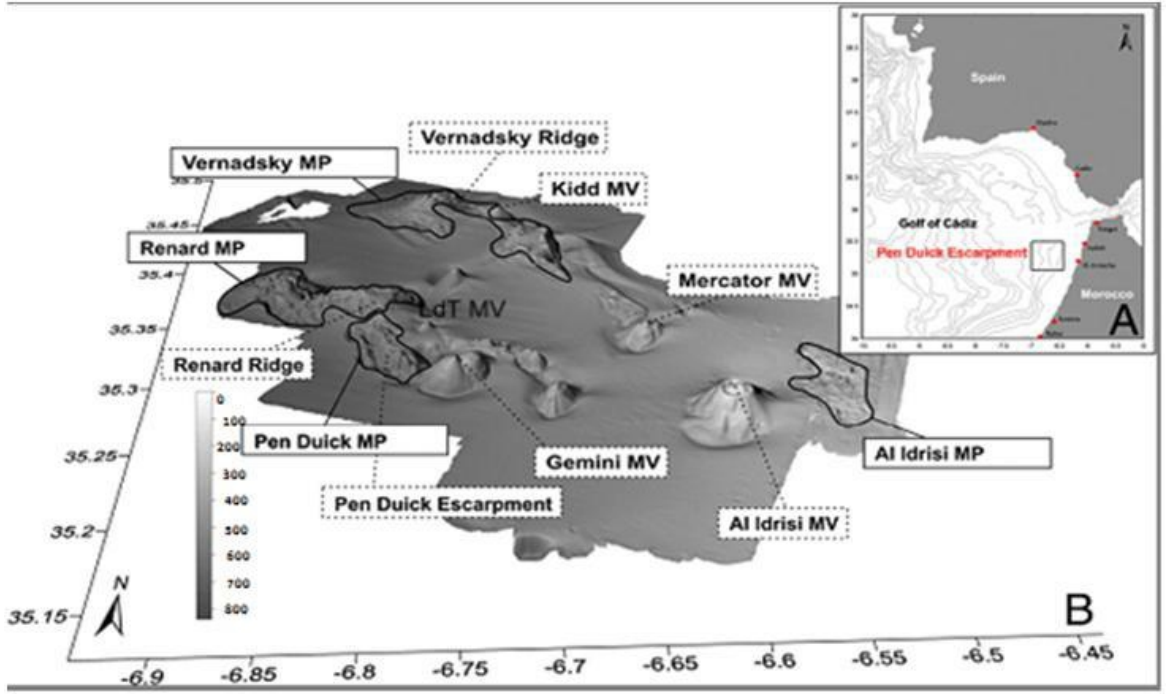

Fig. 1: (A) Localisation de la zone d'étude. (B) carte bathymétrique de la zone d'étude (Foubert \& al.2008).

\section{Matériel et méthode}

Les données utilisées ont été acquises en 2005 au cours de la mission océanographique M-2005 n64PE237, conduite par l'Institut Royal de la Recherche Maritime de Hollande (NIOZ), à bord du navire de recherche hollandais «PELAGIA » (de Haas \& Mienis 2005).

La carotte M2005-12pc $\left(35^{\circ} 18,318^{\prime} \mathrm{N} ; 6^{\circ} 47,012^{\prime} \mathrm{W} ; \mathrm{d}=538 \mathrm{~m}\right)$ a été recueillie à l'aide d'un carottier à piston (Pistoncore) de $1500 \mathrm{~kg}$, de $12 \mathrm{~m}$ de longueur et de $11 \mathrm{~cm}$ de diamètre (fig. 2).

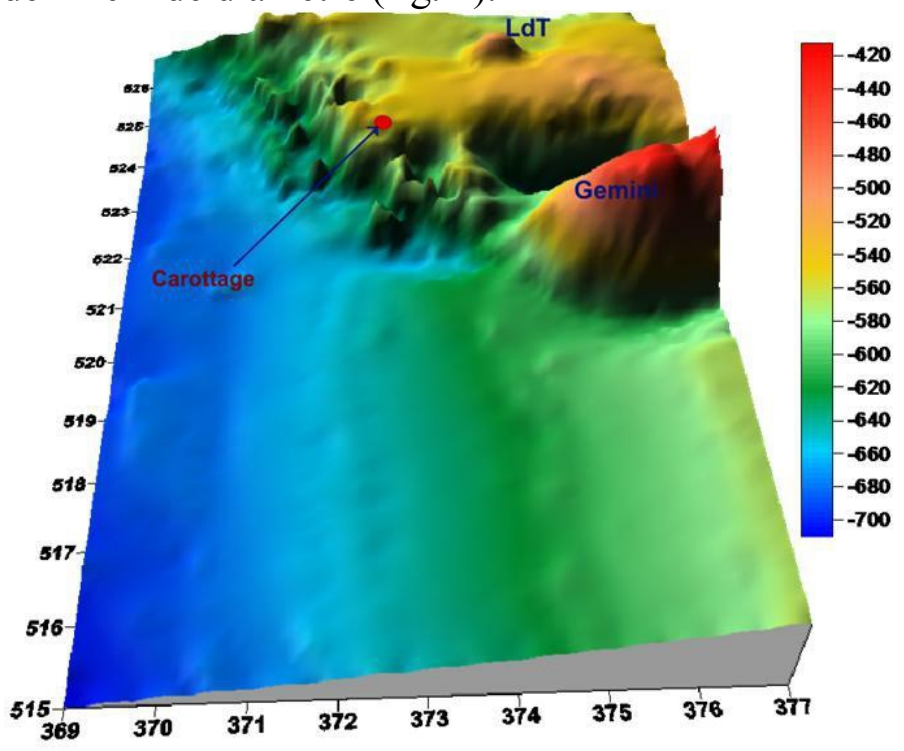

Fig. 2: Carte bathymétrique en 3D de la région d'étude qui représente la zone de carottage 
La carotte a été découpée en segments de $1,1 \mathrm{~m}$. La susceptibilité magnétique a été directement mesurée à bord du navire (De Haas \& Mienis 2005) avec un pas de $2 \mathrm{~cm}$ à l'aide d'un appareil Bartington MS2C avec une boucle de $12 \mathrm{~cm}$ de diamètre. Ensuite, la carotte a été congelée et transportée.

$\mathrm{Au}$ laboratoire, la carotte a été découpée longitudinalement en deux moitiés : l'une de cette dernière a été utilisée pour l'étude, l'autre a été archivée. Après l'observation et la description de la lithologie, la carotte a été introduite et analysée dans un XRF Core Scanner de marque Avaatech, en prenant des photographies pour le dosage de $\mathrm{Ca}$, $\mathrm{Sr}$ et $\mathrm{Fe}$. Pour rassembler les données de XRF utilisées dans cette étude, on a utilisé un électron et $30 \mathrm{kV}$ pour le Strontium. En effet la spectrométrie de fluorescence de rayon $\mathrm{X}$ emploie le processus d'émission de rayon X pour les différents éléments et ce, pour déterminer la composition. Quand les rayons X contactent un électron d'un atome, l'électron absorbe cette énergie pour quitter la coquille. L'espace qui résulte de ceci est rempli par un électron qui vient d'un atome d'une autre coquille. Quand cet électron tombe de nouveau dans une zone à basse force (pour remplir l'espace), il émet la différence d'énergie entre les deux coquilles sous forme de rayonnement électromagnétique. (Brissaud et al. 1988).

Ceci signifie donc que la quantité de rayonnement électromagnétique émis est différente pour chaque élément; elle permet d'analyser la composition de chaque élément par la détection de la quantité de rayonnement spécifique.

Pour l'étude granulométrique et paléontologique, deux échantillons ont été prélevés chaque $5 \mathrm{~cm}$ à l'aide de seringues de $10 \mathrm{ml}$. Un total de 141 échantillons a été prélevé pour chaque groupe. Le premier a été utilisé pour l'analyse granulométrique, le second pour la mesure des isotopes de l'oxygène.

Afin de déterminer la densité et la porosité, les échantillons ont été pesés puis séchés pendant au moins $24 \mathrm{~h}$ et repesés à nouveau. La masse volumique et la porosité ont été calculées à l'aide des équations $\mathrm{F}=\mathrm{Ps} / \mathrm{Pi}$ pour la porosité (où Ps est le poids à sec et Pi le poids initial de l'échantillon); et $\mathrm{r}=\mathrm{Pi} / \mathrm{V}$ pour la masse volumique, où $\mathrm{V}$ est le volume prélevé.

Pour l'étude de la distribution granulométrique (fraction 0,04-2 mm), 80 à $100 \mathrm{mg}$ de sédiment ont été prélevés de chaque échantillon, pesés dans un bécher puis dissous dans $30 \mathrm{ml}$ d'eau. Les béchers ont été placés dans un bain ultrasonique pendant $5 \mathrm{~min}$, tamisés à l'aide d'un tamis de $2 \mathrm{~mm}$ et finalement mesurés à l'aide d'un appareil Coulter LS 230. La composition granulométrique est calculée semi-quantitativement à partir des diffractogrammes.

Le deuxième groupe d'échantillon a été tamisé pour obtenir deux fractions : la première, de diamètre $63-150 \mu \mathrm{m}$ a été archivée alors que la 
deuxième, de diamètre $>150 \mu \mathrm{m}$, a subi un autre tamisage pour l'extraction de la fraction à diamètre de $250-300 \mu \mathrm{m}$. La mesure de $\delta \mathrm{O}^{18}$ a été effectuée pour chaque échantillon sur 5 individus (de 20 initiaux prélevés) de foraminifères planctoniques de l'espèce Globigerinoides ruber, de diamètre de 250-300 $\mu \mathrm{m}$. La teneur isotopique des fluides et des gaz à été mesurée à l'aide d'un appareil Gas Bench II équipé d'un delta + et d'un spectromètre de masse. Chaque échantillon a été mesuré 8 fois, l'opération débutant par le calibrage à l'aide de deux échantillons standard et se terminant avec un autre calibrage. Chaque échantillon est mesuré par rapport à un SMOW (Standard Mean Oceanic Water) avec un écart type de $0,15 \% \delta \mathrm{O}^{18}$.

\section{Résultats et interprétation:}

\section{Faciès et caractéristiques lithologiques et physiques:}

L'analyse au sonar à balayage latéral de l'Escarpement Pen Duick a révélé quatre faciès distincts (Foubert et al. 2008): (i) formations sableuses à silto-argileuses (fig. 3), avec quelques galets (faciès 1); ce faciès a été identifié à la surface des différents monticules de l'Escarpement Pen Duick, (ii) fragments de coraux d'eau froide et de boue sableuse de couleur brunâtre (faciès 2); ce faciès a été reconnu à la surface sommitale de tous les monticules, (iii) croûtes carbonatées et de roche couverte par une couche fine de boue hémi-pélagique (faciès 3 ), et (iv) des dépôts de fragments de coraux d'eau froide (faciès 4 ) issus de lessivage par des courants très érosifs au pied de l'escarpement à pente raide. Dans la carotte étudiée, seuls les faciès 1 et 2 ont été reconnus.

La lithologie dominante est une vase silto-argileuse avec des tâches noires dont certains assez grandes (vers $614 \mathrm{~cm}$ ) et des foraminifères. La couleur est gris jaunâtre à olive grisâtre $(5 \mathrm{Y}-4 / 2)$ passant parfois au brun jaunâtre mat $(10 \mathrm{Y}-4 / 3)$ vers $150-175 \mathrm{~cm}$, gris $(5 \mathrm{Y}-4 / 1)$ vers $175-226 \mathrm{~cm}$; noire olive à olive grisâtre $(437-595 \mathrm{~cm})$ ou bien olive grisâtre à olive foncé (625-705 cm).

Ce faciès montre des sables fins $(132-175 \mathrm{~cm})$, des niveaux silteux plus grossiers $(205-226 \mathrm{~cm}, 425-437 \mathrm{~cm})$ et des fragments de coraux du genre Desmophyllum $(145-148 \mathrm{~cm}$ ). Des débris carbonatés (gastéropodes, bivalves) sont rencontrés dans les intervalles $132-150 \mathrm{~cm}, 285-305 \mathrm{~cm}$ et $487-500 \mathrm{~cm}$. 


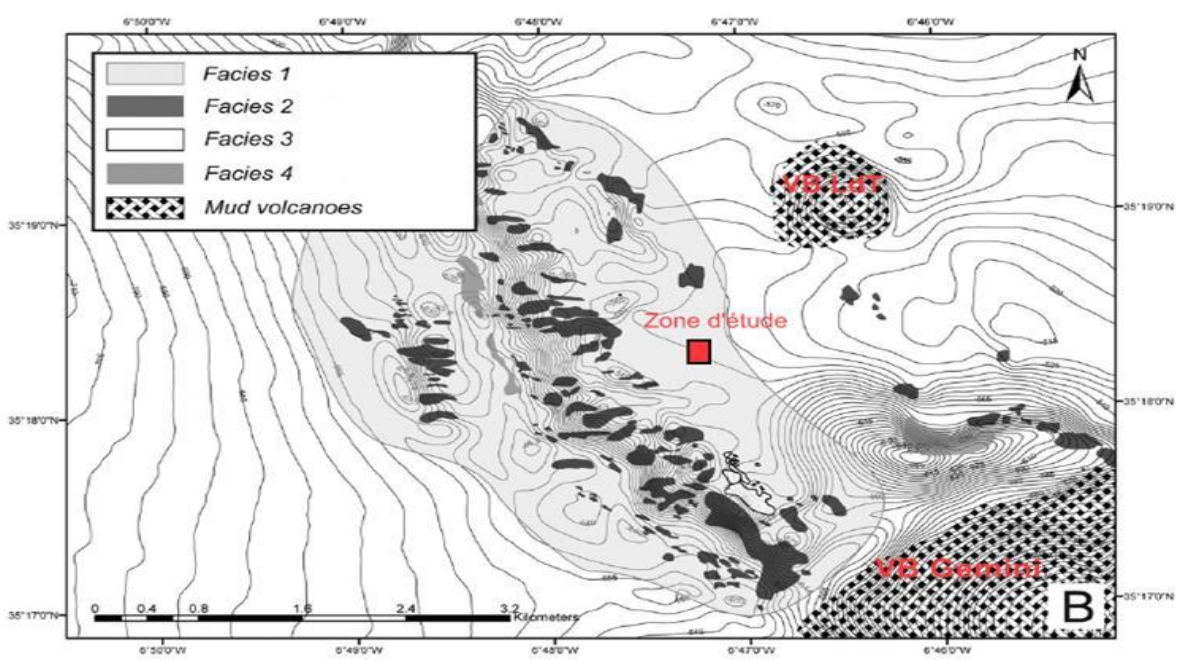

Fig. 3: Carte bathymétrique de la zone d'étude couplée à la cartographie sonar, Faciès 1: Sable à vase Silto-argileuse, Faciès 2: Coraux d'eau froide Faciès 3: Croûtes carbonatés, Faciès 4: Structures lessivés (scoured structures) (Foubert \& al.2008).

Le profil de distribution granulométrique (fig. 4) montre une dominance de la fraction plus fine $(<63 \mu \mathrm{m})$ dans presque toute la carotte d'un pourcentage supérieur à $50 \%$. Des changements brusques de granulométrie sont observés vers $135-150 \mathrm{~cm}$ et $490 \mathrm{~cm}$ et sont traduits par une chute de la fraction fine à $23 \%$ et une augmentation de fractions grossières de $63-250 \mu \mathrm{m}$ et $250 \square \mathrm{m}-1 \mathrm{~mm}$ à $44 \%$ et $56 \%$ respectivement. Des niveaux à fraction 1-2 $\mathrm{mm}$ sont détectés à $130-150 \mathrm{~cm}, 210-225 \mathrm{~cm}$, $335-340 \mathrm{~cm}, 500 \mathrm{~cm}$ et á $655-680 \mathrm{~cm}$.

Les mesures de la porosité montrent que celle-ci est élevée (73\%) au sommet de la carotte et diminue plus en profondeur. Les valeurs maximales de la porosité $(\sim 59 \%)$ relevées vers $150 \mathrm{~cm}$ et $220 \mathrm{~cm}$, correspondent aux couches de sable décrites ci-dessus.

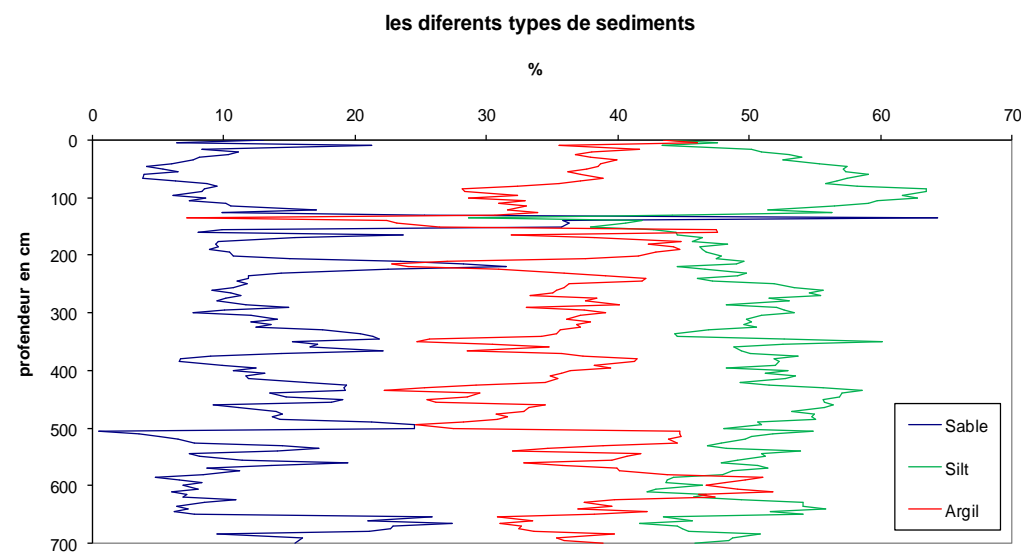

Fig. 4: Distribution granulométrique de la carotte M2005-12. 
La susceptibilité magnétique générale fluctue autour de $30 \mathrm{cgs}$ en moyenne jusqu'à $250 \mathrm{~cm}$ de profondeur, puis elle augmente notablement plus en profondeur, fluctuant autour de $35 \mathrm{cgs}$ (fig. 5). Les valeurs minimales à $65,375,485,600$ et $705 \mathrm{~cm}$ coïncident avec les frontières des sections composant la carotte et sont des artefacts liés à la procédure de mesure. Le pic aigu de 42 cgs au sommet de la carotte correspondrait à un enrichissement des sédiments de surface par l'oxyde de fer et de manganèse. La courbe suit approximativement celle de la granulométrie à fraction fine ; les valeurs minimales à 150 et $220 \mathrm{~cm}$ correspondent aux couches de sable non magnétique.

\section{Géochimie ( $\mathrm{Ca}, \mathrm{Fe}, \mathrm{Sr}$ et $\operatorname{rapport} \mathrm{Ca} / \mathrm{Sr})$ :}

La carotte montre des valeurs de calcium moyennes de $8 \times 104$ à 105 cps avec des pics à $160-170 \mathrm{~cm}, 350-410 \mathrm{~cm}$ et à $560-620 \mathrm{~cm}$. La variation du contenu en fer est en sens inverse à celle du calcium, sauf pour les premiers centimètres mais généralement avec des valeurs modérées (inférieures à $45 \times 103 \mathrm{cps}$ ) (fig. 6).

Le contenu en strontium montre des valeurs qui fluctuent autour de $6 \times 103 \mathrm{cps}$, vers $20 \mathrm{~cm}, 130-190 \mathrm{~cm}, 390 \mathrm{~cm}$ et $580-630 \mathrm{~cm}$, qui traduisent un contenu en coraux important.

Les valeurs du rapport $\mathrm{Ca} / \mathrm{Sr}$ fluctuent autour d'une moyenne de 15 . Les intervalles de profondeur $126-153 \mathrm{~cm}, 212-225 \mathrm{~cm}$ et $338-360 \mathrm{~cm}$ montrent un faible rapport de $\mathrm{Ca} / \mathrm{Sr}$ lié à de nombreux débris de coraux (description de la carotte) (fig. 6).

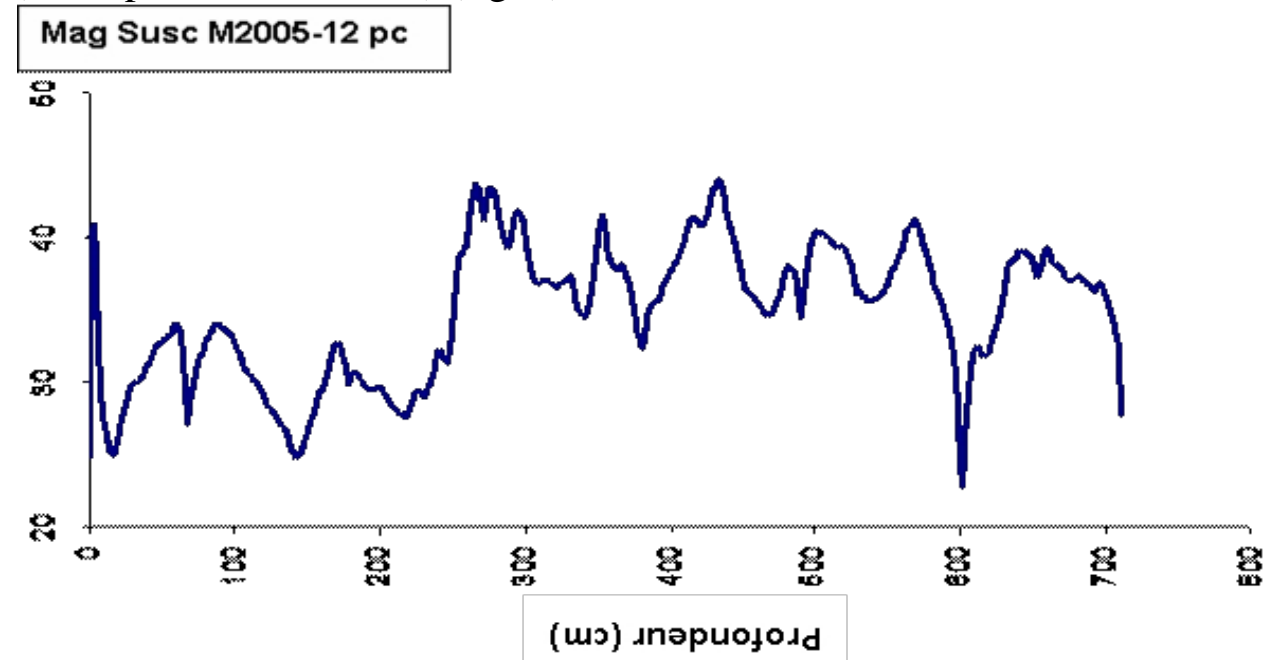

Fig. 5 : Variation de la susceptibilité magnétique en fonction de la profondeur. Cruise report R.V Pelagia cruise M2005 (64PE237) 

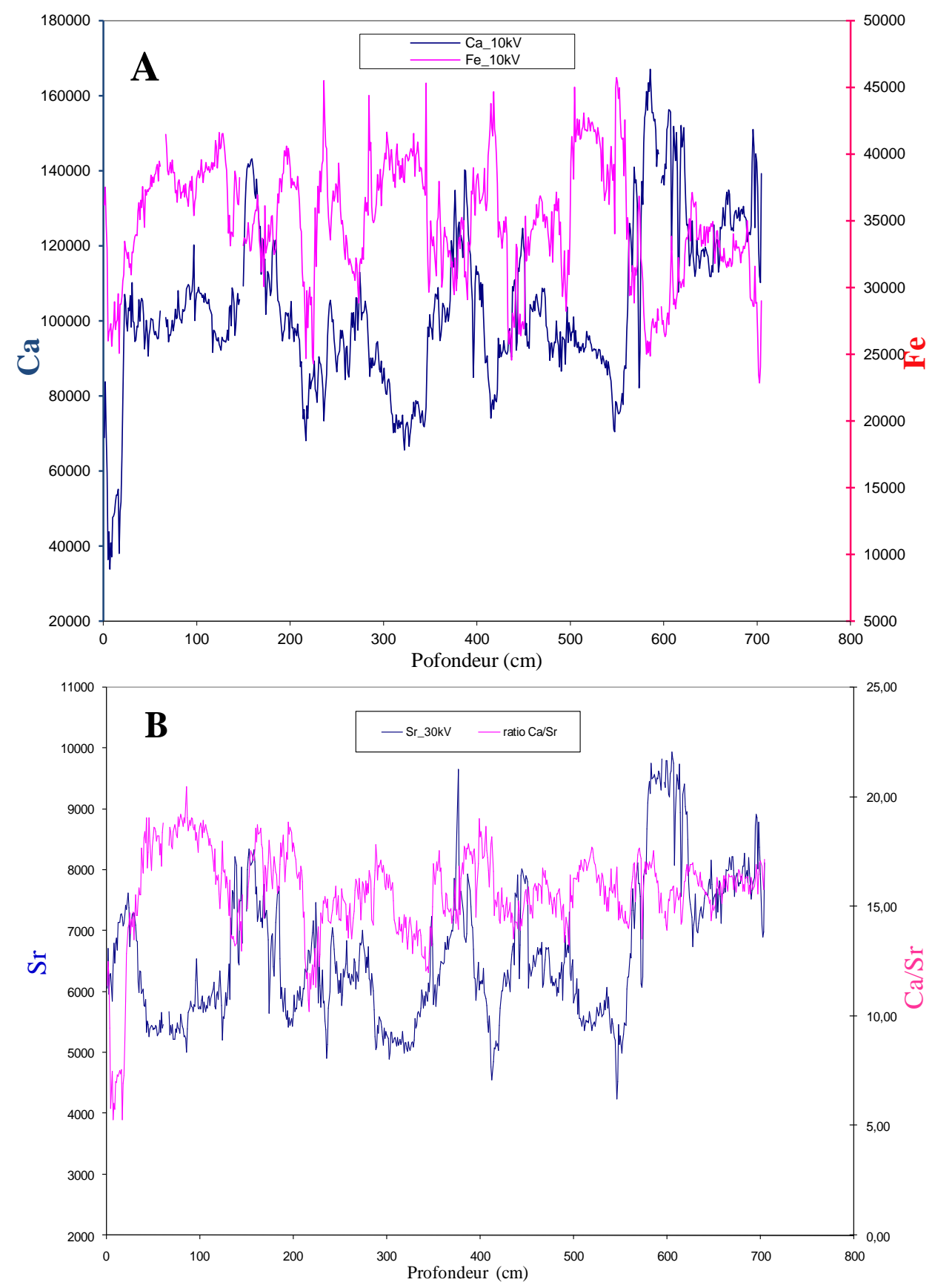

Fig. 6: Géochimie de la carotte. (A) Répartition du Fe et du Ca. (B) Le rapport $\mathrm{Ca} / \mathrm{Sr}$ : Cruise report R.V Pelagia cruise M2005 (64PE237) 


\section{Isotopes stables de l'oxygène}

Le profil de $\delta \mathrm{O}^{18}$ pour Globigerinoides ruber montre des valeurs fluctuant autour de la valeur moyenne $1 \%$. Pour détailler, on assiste à valeurs maximales absolues à $130 \mathrm{~cm}$ (valeur 1,8\%), $235 \mathrm{~cm}(1,62 \%$ ), 340 $\mathrm{cm}(1,61 \%)$ et $485 \mathrm{~cm}(1,64 \%)$, et un maximum relatif vers $560 \mathrm{~cm}$ $(1,21 \%)$. Alors que les valeurs minimales absolues apparaissent vers 0,25 $\mathrm{cm}$ $(-0,45 \%)$ et $180 \mathrm{~cm}(-0,53 \%)$ (fig. 7$)$.

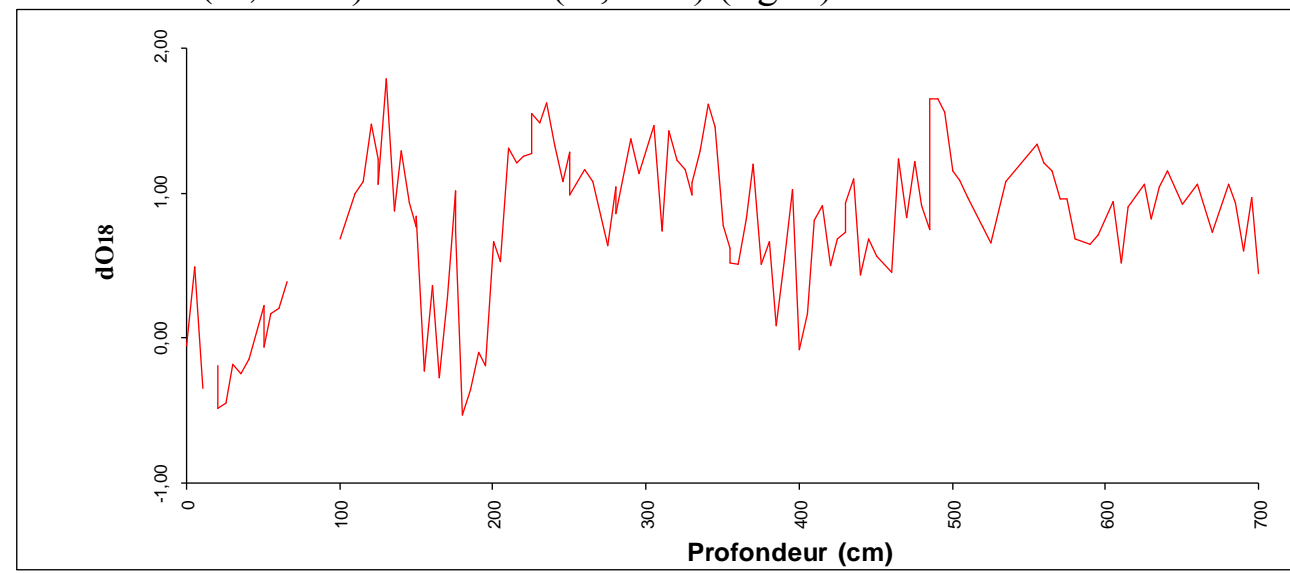

Fig. 7 : Valeurs des $\delta \mathrm{O}^{18}$ pour les Foraminifères Planctoniques (G. ruber)

\section{Discussion}

\section{Environnements de dépôt}

Pour les sédiments de surface $(0-130 \mathrm{~cm})$, le passage progressif du gris jaunâtre vers l'olive grisâtre reflète une variation de la composition minéralogique, la susceptibilité magnétique traduit cette variation par des valeurs élevées au sommet de la carotte qui correspondent à un enrichissement des sédiments de surface en oxydes de fer et de manganèse. Plus bas, les valeurs de la susceptibilité magnétique deviennent faibles (intervalle $10-250 \mathrm{~cm}$ ) alors que la lithologie montre des débris carbonatés et des taches noires. Le diagramme XRF montre un rapport $\mathrm{Ca} / \mathrm{Sr}$ élevé; ce qui indique une réduction de la proportion des fragments de coraux dans les débris. La répartition granulométrique dominante est de type silto-argileuse à argilo-silteuse.

La partie intermédiaire de la carotte $(130-230 \mathrm{~cm})$ se caractérise par un changement brusque de la couleur et de la granulométrie, surtout vers 130 $\mathrm{cm}$ et $220 \mathrm{~cm}$, où la couleur passe de l'olive grisâtre vers le brun jaunâtre. L'étude granulométrique montre des éléments plus grossiers $(250 \mu \mathrm{m}-1 \mathrm{~mm})$ correspondant à des sables fins. Des valeurs importantes de $\mathrm{Ca}$ et $\mathrm{Sr}$ sont enregistrées à cette profondeur $(150 \mathrm{~cm})$; ce qui s'explique par la présence des fragments de coraux (Desmophyllum) et des débris carbonatés sachant que le strontium est assimilé par les coraux. 
Dans la section profonde de la carotte $(230-705 \mathrm{~cm})$, la granulométrie est identique à celle de la superficie de dominance silto-argileuse à argilosilteuse (fraction fine $<63 \mu \mathrm{m}$ ). Le saut rapide de la susceptibilité magnétique vers des valeurs plus élevées caractérise une grande réserve en matériel terrigène. Le faible rapport de $\mathrm{Ca} / \mathrm{Sr}$ serait donc lié à la présence des débris carbonatés (Gastéropodes, Bivalves).

\section{Variations de $\delta \mathrm{O}^{18}$ :}

Les 4 valeurs maximales absolues observées à $130 \mathrm{~cm}\left(\delta \mathrm{O}^{18}\right.$ $=1,8 \%), 235 \mathrm{~cm}\left(\delta \mathrm{O}^{18}=1,62 \%\right), 340 \mathrm{~cm}\left(\delta \mathrm{O}^{18}=1,61 \%\right)$ et $485 \mathrm{~cm}\left(\delta \mathrm{O}^{18}\right.$ $=1,64 \%$ ) et le maximum relatif vers $560\left(\delta \mathrm{O}^{18}=1,21 \%\right)$ correspondent à des périodes chaudes ou à des foraminifères de périodes chaudes. Les valeurs minimales absolues apparaissent vers $0,25 \mathrm{~cm}\left(\delta \mathrm{O}^{18}=-0,48 \%\right)$ et $180 \mathrm{~cm}$ $\left(\delta \mathrm{O}^{18}=-0,53 \%\right)$, correspondent à des périodes froides ou à des foraminifères de périodes froides (Rozanski et al. 1990).

En l'absence de datations radiométriques (en cours), l'estimation des âges peut être tentée à travers la comparaison avec la courbe $\delta \mathrm{O}^{18}$ de la carotte M39008 forée plus au Nord, vers $36^{\circ} 22,9^{\prime \prime} \mathrm{N} ; 7^{\circ} 4,6^{\prime} \mathrm{W}$ (Cacho et al. 2001) basée sur G. bulloides (fig. 8). La comparaison pic-à-pic (fig. 9) suggère que :

- Les faibles valeurs de $\delta \mathrm{O}^{18}$ à $0-50 \mathrm{~cm}$ et à $150-200 \mathrm{~cm}$ représentent les eaux chaudes de la période Holocène (0-11 ka BP).

- La transition de la dernière période glaciaire à l'Holocène serait enregistrée au-dessous de la couche de sables à $220 \mathrm{~cm}$.

- Les valeurs maximales de $\delta \mathrm{O}^{18}$ à $230 \mathrm{~cm}$ et à $340 \mathrm{~cm}$ représentent l'intervalle froid du Younger Dryas vers $11-13 \mathrm{ka}$ BP, alors que les valeurs de $\delta \mathrm{O}^{18}$ à $\sim 1,5 \%$ o se corrèlent bien avec celles de cet intervalle pour la carotte M39008;

- Au-dessous de $230 \mathrm{~cm}$, les valeurs de $\delta \mathrm{O}^{18}$ demeurent entre 0,5 et $1,4 \%$ qui correspondent bien aux valeurs trouvées entre 11 et $15 \mathrm{ka}$ BP dans la carotte M39008; les valeurs normales et maximales trouvées pour la dernière période glaciaire (2-2,5\%o) ne figurent pas dans M2005-12pc.

Les valeurs anormales de $\delta \mathrm{O}^{18}$ autour de $130 \mathrm{~cm}$ dans la carotte M2005-12pc correspondent aux foraminifères d'une période climatique plus froide. Ces valeurs correspondent aux décharges plus grossières (sables) au sein de la carotte. Ceci suggère par conséquent que les foraminifères ont été transportés et déposés à l'intérieur des couches sédimentaires. 


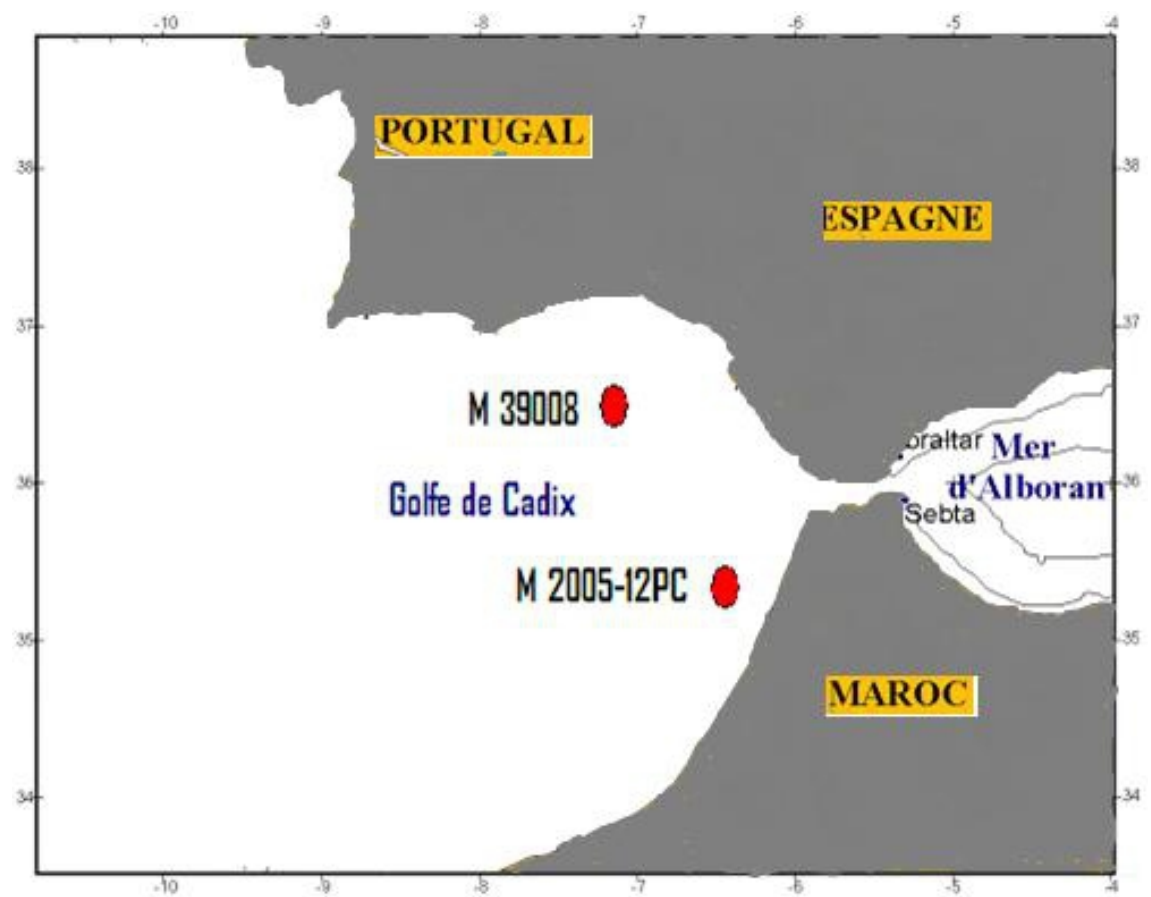

Fig. 8: Localisation des carottes M39008 et M2005-12pc dans le Golfe de Cadix.

Sur le plan environnemental, l'interprétation de la variation de la susceptibilité magnétique de la granulométrie et de $\delta \mathrm{O}^{18}$ associés suggèrent que :

- L'Holocène, représenté par l'intervalle 0-220 cm (ou 0-270 cm ?), est caractérisé par une sédimentation hémi-pélagique interrompue par une forte décharge de sédiments probablement glaciaires.

- La dernière période glaciaire est représentée par l'intervalle 220$700 \mathrm{~cm}$ (ou 270-700 cm) qui se caractérise par un taux de sédimentation plus élevé qu'au cours de l'Holocène en raison d'une plus grande réserve en matériel terrigène à grain fin. La contribution terrigène plus grande est reflétée par les valeurs plus élevées de la susceptibilité magnétique dans cet intervalle. 


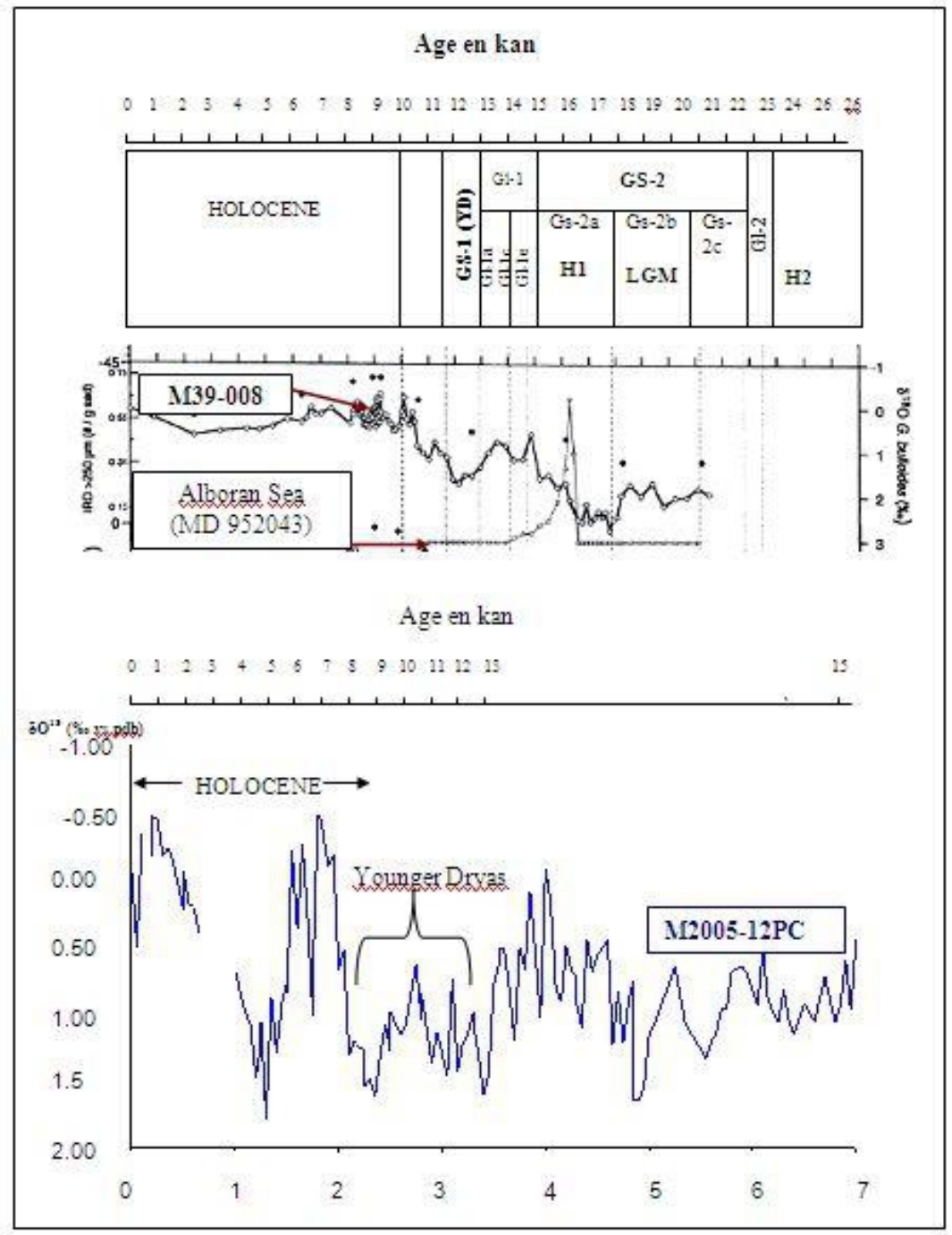

Fig. 9 : Comparaison des valeurs de $\delta \mathrm{O}^{18}$ entre la carotte $\mathrm{M} 2005-12 \mathrm{pc}$ (G.ruber) et la carotte M39008 (G. bulloides).

La perturbation trouvée dans les valeurs isotopiques de $\delta \mathrm{O}^{18}$ au niveau des sédiments déposés autour de $130 \mathrm{~cm}$ et $220 \mathrm{~cm}$ se traduit par un changement dans la région qui se matérialise par la présence d'un matériel sableux qui caractérise un courant de forte énergie (Nelson et al. 1999). Ces perturbations pourraient résulter d'une modification de la circulation océanique manifestée par un courant froid responsable sur les dépôts sableux de l'âge glaciaire ou par une période d'érosion importante dont les sédiments qui sont probablement déposés par l'oued Loukkos. 


\section{Conclusion}

L'Escarpement Pen Duick est caractérisé par une histoire géologique complexe et par la présence des volcans de boue.

Les sédiments récoltés dans la carotte (M2005-12PC) qui datent probablement de l'Holocène sont représentés dans l'intervalle $0-220 \mathrm{~cm}(-270$ cm ?) par des dépôts hémi-pélagiques interrompus par deux grandes masses sédimentaires caractérisant des sédiments glaciaires. La lithologie dans cet intervalle montre une dominance des sédiments silto-argileux qui sont intercalés par des débris carbonatés et des taches noires. Ces dernières sont probablement causées par la diagenèse.

L'intervalle 220-700 $\mathrm{cm}$ (ou 270-700 cm) représente la dernière période glaciaire qui se caractérise par un taux de sédimentation plus important que dans l'Holocène en raison d'une réserve plus grande en matériel terrigène fin. La contribution terrigène importante est reflétée par les valeurs plus élevées de la susceptibilité magnétique trouvées dans cet intervalle.

\section{References:}

1. Brissaud, I., Houdayer, A., Vivier, G., Keller, F. (1988). Analyse comparative de roches par fluorescence $X$ induite par rayonnement de basse énergie ( pp. 1-10).

2. Cacho, I., Grimalt, J. O., Canals, M., Sabffi, L., Shackleton, N. J., Schonfeld, J. \& Zahn, R. (2001). Variability of the western Mediterranean Sea surface temperature during the last 25,000 years and its connection with the Northern Hemisphere climatic changes (pp. 16, 1, 40-52). Paleoceanography.

3. De Hass, H., Mienis, F \& Shipboard Scientific Crew. (2005). Cold water corals and carbonate mound formation at the Pen Duick Escarpment (Gulf of Cadiz) and Rockall Band. Cruise report R.V. Pelagia cruise M2005 (64PE237): Ed . Royal NIOZ, 80 p.

4. De Hass, H. (2006). Seismic and sedimentological investigations of the carbonate mounds and mud volcanoes at the Pen Duick Escarpment and SE Gulf of Cadiz, Cruise report R.V.Pelagia cruise M2006 (64PE253).

5. De Hass, H., Shipboard Scientific party. (2006). Seismic and sedimentological investigations of the carbonate mounds and mud volcanoes at the Pen Duick Escarpment and SE Gulf of cadiz. Cruise report R.V. Pelagia cruise M2006 (64PE253).

6. Foubert, A., Depreiter, D., Beck, T., Maignien, L., Pannemans, B., Frank, N., Blamart, D. \& Henriet, J. (2008). Carbonate mounds in a mud volcano province off north-west Morocco: Key to processes and controls. Mar Géol. (pp. 248, 74-96). 
7. Hamoumi, N., Henriet, J., Kenyon, N. \& Suzyumov A. E. (2005). Geosphere-Biosphere coupling processes: the TTR interdisciplinary approach towards studies of the European and North African margins.

8. International Conference and Post-Cruise Meeting of the TrainingThrough-Research Programme Marrakech, Morocco 2 - 5 February. (2005). Intergovernmental Oceanographic Commission: Workshop Report (pp. 10, 71, 197).

9. Kenyon, N. H., Ivanov, M .K., Akhmetzhanov, A.M. \& Akhmanov, G. G. (2003). Interdisciplinary Geoscience research on the North East Atlantic margin, Mediterranean sea and Mid-Atlantic ridge. Preliminary results of investigations during the TTR-12 cruise of RV Professor Logachev, June-August 2002: Intergov. Oceanogr. Commission, Techn. Ser, (pp 67, $112+35)$.

10. Maldonado,. A., Somoza, A. \& Pallarés L. (1999). The Betic orogen and the Iberian African boundary in the Gulf of Cadiz: geological evolution (central North Atlantic). Mar. Geol (pp 9-43, 155).

11. Medialdea, T., Vegas, R., Somoza, L., Vázquez, J. T., Maldonado, A., Díaz del Río V., Maestro, A., Córdoba, D. \& Fernández-Puga M .C. (2004). Structure and evolution of the "Olistostrome" complex of the Gibraltar Arc in the Gulf of Cádiz (eastern Central Atlantic): evidence from two long seismic cross sections. Mar. Geol (pp 173198, 209).

12. Nelson, C. H., Baraza, J., Maldonaldo, A., Rodero, J., Escutia, C. \& Barber J. H. (1999). Influence of the Atlantic inflow and Mediterranean outflow currents on Late Quaternary sedimentary facies of the Gulf of Cadiz continental margin. Mar. Geol (pp 99-105, $155)$.

13. Pinheiro, L. M., Ivanov, M. K., Sautkin, A., Akhmanov, G., Magalhaes V. H., Volkonskava, A., Monterio, J. H., Somoza, L., Gardner, J., Hamoumi, N \& Cunha, M. R. (2003). Mud volcanism in the Gulf of Cadiz: results form the TTR-10 cruise. Mar. Geol (pp 131-151, 195)

14. Rozanski ,K., Gonfiantini, R. (1990). Les isotopes de l'environnement facilitent l'étude de nos climats. AIEA Bulletin Perspectives, 4/1990.

15. Samoza, L., Díaz-del-Río, V., León, R., Ivanov, M., Fernández-Puga, M.C., Gardner, J.M., Hernández-Molina, F.J., Pinheiro, L.M., Rodero, J., Lobato, A., Maestro, A., Vázquez, J.T., Medialdea, T., Fernández-Salas, L.M. (2003). Seabed morphology and hydrocarbon seepage in the Gulf of Cádiz mud volcano area: Acoustic imagery, multibeam and ultra-high resolution seismic data. Marine Geology (pp 153-176 195). 
16. Stadnitskaia, A., Ivanov, M. K., Blinova, V., Kreulen, R. \& van Weering T.C.E. (2006). Molecular and carbon isotopic variability of hydrocarbon gases from mud volcanoes in the Gulf of Cadiz, NE Atlantic. Mar. Petr. Geol (pp 23, 281-296).

17. Van Rensbergen P.V., Depreiter, D., Ivanov, M., and Shipboard Scientific Party of the TTR-12 Cruise. (2003). El Araiche mud volcano field. In: Kenyon, N. H., Ivanov, M. K., Akhmetzhanov A. M. \& Akhmanov, G. G. (eds) - Interdisciplinary Geoscience research on the North East Atlantic margin, Mediterranean sea and Mid-Atlantic ridge. Preliminary results of investigations during the TTR-12 cruise of RV Professor Logachev, June-August 2002. Intergov. Oceanogr. Commission, Techn. Ser (pp 43-50, 67).

18. Van Rensbergen, P.V., Depreiter, D., Pannemans, B., G. M, V Rooij, D., Marsset, B., Blinova, Ivanov, M., Rachidi, M., Pinheiro, V. M. L., Cunha, M \& Henriet J. P. (2005). The El Arraiche mud volcano field at the Moroccan Atlantic slope, Gulf of Cadiz. Mar. Geol (pp 117, 219).

19. Van Rooij, D., Blamart, D., De Mol, L., Mienis, F., Pirlet, H., Wehrmann, L. M., Barbieri, R., Maignien, L., Templer, S. P., de Haas, H., Hebbeln, D., Frank, N., Larmagnat, S., Stadnitskaia, A., Stivaletta, N., Van Weering, T., Zhang, Y., Hamoumi, N., Cnudde V., Duyck, P., Henriet, J. P. (2011). Cold-water coral mounds on the Pen Duick Escarpment, Gulf of Cadiz: The Microsystems project approach, Mar Géol (pp 102-117, 282). http://www.nioz.nl/public/dmg/rpt/crs/64pe237.pdf 